\title{
Del modelo conservacionista al patrimonio territorial y el turismo en Costa Rica
}

Omar Barrantes Sotela

Lilliam Quirós Arias

\section{SciELO Books / SciELO Livros / SciELO Libros}

BARRANTES SOTELLA, O., and QUIRÓS ARIAS, L. Del modelo conservacionista al patrimonio territorial y el turismo en Costa Rica. In: MARAFON, G. J., FACCIOLI, M., and SÁNCHEZ, M. A., ed. Patrimônio, território e turismo no Brasil, Costa Rica e Itália [online]. Rio de Janeiro:

EDUERJ, 2020, pp. 9-38. ISBN: 978-65-00-03032-7.

https://doi.org/10.7476/9786500030327.0002.

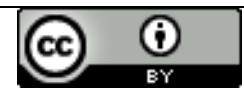

All the contents of this work, except where otherwise noted, is licensed under a Creative Commons Attribution 4.0 International license.

Todo o conteúdo deste trabalho, exceto quando houver ressalva, é publicado sob a licença Creative Commons Atribição 4.0.

Todo el contenido de esta obra, excepto donde se indique lo contrario, está bajo licencia de la licencia Creative Commons Reconocimento 4.0. 


\section{Del modelo conservacionista al patrimonio territorial y el turismo en Costa Rica}

Omar Barrantes Sotela ${ }^{1}$

Lilliam Quirós Arias ${ }^{2}$

\section{Introducción}

Costa Rica es mencionado de manera continua como un modelo exitoso de la conservación del medio ambiente (Rosendal \& Schei, 2014). Cuenta con un sistema de conservación que abarca un $25.25 \%$ de su territorio continental que comprende $51.100 \mathrm{Km}^{2}$ y que mantiene bajo diferentes categorías de manejo mediante rango de ley de la República (Sinac, 2017).

Según Evans (1997), el Sistema de Áreas Silvestres Protegidas de Costa Rica es uno de los más complejos de Latinoamérica y protege la gran biodiversidad del país. Datos de las colecciones del Museo Nacional de Costa Rica reportan más de 850 especies de aves, 220 especies de reptiles, entre 160 especies de anfibios, 240 especies de mamíferos (la mayoría del orden Chiroptera) y más de 130 especies de peces de agua dulce. También se cuenta con registros de alrededor de 9000 especies de plantas vasculares ( $5 \%$ de la biodiversidad del planeta) de las cuales se pueden contabilizar más de 1200 especies de orquídeas y 1200 especies diferentes de árboles. Lo anterior, sin olvidar la gran cantidad

\footnotetext{
Académico Escuela de Ciencias Geográficas, Universidad Nacional, Costa Rica. Correo electrónico: omar.barrantes.sotela@una.ac.cr

2 Académica Escuela de Ciencias Geográficas, Universidad Nacional, Costa Rica. Correo electrónico:lquiros@una.cr
} 
y variedad de insectos descritos. Solo el INBio $^{3}$ indica haber contabilizado más de 15 mil especies de insectos.

Hall (1985) ofrece una de las explicaciones por las cuales el país cuenta con una gran biodiversidad; indica que por su origen geológico es el único lugar en el planeta que es tanto interoceánico como intercontinental. Es un puente y filtro biogeográfico, que en conjunto con sus características de topografía y clima favorecen la diversificación de especies. Por lo tanto, es una zona de convergencia de interés para el estudio del desplazamiento de especies entre los hemisferios norte y sur del continente americano. Entre los últimos casos reportados de este fenómeno en el 2015 y 2017 son los avistamientos del perro de arbusto (Speothos venaticus) y la rata de árbol de Rufous (Diplomys labilis), aunque su nuevo patrón de distribución se asocia más a la perdida de hábitat.

Su gran patrimonio territorial expreso en su fisonomía y gran riqueza de suelos agrícolas posibilitaron flujos migratorios de la depresión tectónica central (Valle Central) del país hacia las zonas periféricas; de ahí que la colonización agrícola impactó fuertemente esa base de recursos, propiciando una gran degradación ambiental. Revertir este proceso de degradación de recursos, y el surgimiento de un nuevo paradigma en la relación sociedad-naturaleza, conllevó a la adopción de prácticas ambientales para recuperar esas áreas del territorio; que fueron impulsadas por políticas que tenían como objetivo, colonizar amplias zonas del territorio nacional (Sandner, 1964).

El turismo y especialmente el ecoturismo que se empieza a desarrollar más claramente a partir de los años 1980's, con antecedentes a inicios de los años 1950's, entendió y aprovechó esta gran posibilidad de contar con un patrimonio natural, nutrido de la mayor biodiversidad en el mundo. Con un pasado histórico que favoreció al país de una democracia consolidada y un desarrollo educativo y condiciones de salud por encima de otras naciones en la región. Bajo este contexto, se inicia un proyecto - país en el cual la conservación y el turismo están estrechamente vinculados. La promoción a nivel internacional y las facilidades de los medios de comunicación en el momento actual, facilitan incrementar la visitación turística año a año. Aunque la principal motivación para visitar el país son las actividades de sol y playa; una gran cantidad de actividades vinculadas directamente con el aprovechamiento de los recursos naturales se encadenan a esta motivación inicial como la visitación a los volcanes,

3 Información según inventario antes de su cierre “administrativo” en el 2015. 
observación de la flora y la fauna, visita a las áreas naturales protegidas, entre otras. Los datos registrados por el Instituto Costarricense de Turismo (ICT) 2018, muestran que las divisas aportadas por el turismo en Costa Rica superan actividades tradicionales como café y banano, que en el año 2017 estas representan 3864 de las divisas, mientras que el café aporta 305,3 y el banano unos 1042,2 millones en dólares americanos.

Sí se valoran los principios de conservación de la biodiversidad, los argumentos anteriores pueden ser muy convincentes para reforzar la idea de que los espacios naturales en Costa Rica deben ser conservados. Pero es importante preguntar ¿Sí siempre fue así? ¿Cómo se desarrolló el modelo de conservación en Costa Rica? ¿Es exitoso o no? ¿Se beneficia la actividad turística del patrimonio natural? ¿Hacia dónde va el modelo de conservación y turismo para garantizar la sostenibilidad de ambos derroteros? Estas y otras preguntas deben hacerse para evaluar el estado del patrimonio natural y sobre todo orientar nuevos esfuerzos relacionados con la protección de los recursos naturales y de la cual se benefician actividades económicas como el turismo de relevancia para el país.

\section{Antecedentes}

Según Boza (2015), la llegada de los españoles marca el inicio del deterioro ambiental del país. Se estima por Tenorio (1988), que en la etapa de la conquista la población no superaba los 27200 habitantes y la tasa de deforestación apenas llegaba al 1\%. Con el desarrollo de las actividades económicas de subsistencia en la colonia inician los primeros asentamientos humanos que se consolidan para finales de 1706.

Durante ese período existe muy poca evidencia sobre los impactos ambientales que generaron los habitantes. Según Boza (2015, p. 27), las primeras ordenanzas de protección ambiental se dictaron a la protección de las costas en 1754. Se establece un área de uso público con una extensión de una milla a lo ancho y que sigue el contorno de los litorales. Una disposición que tenía una orientación estrictamente de defensa militar para la protección de los navíos y embarcaciones. El propósito original cambiaría en el Decreto Ley 162 de 1828, en la que se reservan los terrenos baldíos con utilidad común para la marina, pesquería y elaboración de sales, hasta una milla sobre las costas del mar inclusive a favor de estas actividades. 
Un caso similar correspondió evitar los incendios forestales en 1775, por lo que se prohibió tal práctica por el gobernador español Juan Fernández de Bobadilla (Fournier, 1991). Aunque este tipo de regulación es asociado más a una práctica de manejo agrícola y forestal, con ello se aseguró la permanencia de importantes áreas boscosas alrededor de los principales poblados del área central del país. Aunque, refleja los principios e intereses de protección utilitarista de estas zonas.

En el año de 1800, debido a la lenta evolución de la ocupación del suelo, la población del Valle Central ascendía alrededor de 52000 habitantes y solo el 7.7\% del territorio estaba deforestado (Meléndez-Chaverri, 1977). En 1830, las necesidades de consumo de los países europeos por el algodón, café y tabaco se incrementaron y su alta demanda favoreció el auge de la actividad agrícola, en especial el cultivo de café. Con esta nueva dinámica se establecieron nuevas rutas de exportación y se promovió la primera gran expansión agrícola y migratoria costarricense. Se debe destacar que la mayoría de las fincas cafetaleras controladas por las familias no tenían una gran extensión y se manejaban de manera responsable por sus propietarios, al contrario de las grandes plantaciones de monocultivo en manos de los latifundistas (Evans, 1997).

Granados, C. (2004, p. 30) menciona que a partir de 1850 las exportaciones del grano se aceleran, reestructurando la economía costarricense radicalmente, orientándola al mercado internacional. El café marca la historia agroexportadora del país, ocupando las tierras más accesibles y productivas, reemplazando la producción de alimentos básicos y la ganadería en el Valle Central. Este mismo autor señala que "Desde 1840, el próspero negocio cafetalero induce a una deforestación, cuyas consecuencias debieron haberse hecho sentir".

Un aspecto interesante de mencionar es como la génesis de la cultura costarricense ocurre del proceso de mestizaje entre poblaciones europeas, indígenas, afrodescendientes y chinas. Además, fue proceso que se desarrolló de forma aislada del resto del istmo centroamericano por las difíciles condiciones de acceso que presentaba Costa Rica en la colonia. Además, los principales patrones de conducta social fueron acentuándose con el pasar de los años, en los que se mantuvo una conciencia y la creencia arraigada en el uso razonable de los recursos; en esta perspectiva todos los pueblos contribuyen a la diversidad y riqueza de las civilizaciones y culturas. Es así como menciona Sarkar (2010), que indica la diversidad cultural y la biodiversidad están estrechamente relacionadas. 
Meléndez (2004), en su artículo “Aportes geográficos al imaginario costarricense en el siglo XIX”, señala que en el proceso de construcción de la nacionalidad costarricense la geografía ha jugado un papel significativo. $\mathrm{Ha}$ contribuido a la construcción de ciertas imágenes que identifican el ser costarricense, que se han venido modificando con el tiempo; Territorio, condiciones biogeográficas, homogeneidad racial, educación, modo de vida y situación política”. Los cuales han sido pilares fundamentales en el sentir de esta comunidad imaginada, algunos de los cuales surgieron desde los primeros años de vida independiente y han perdurado hasta nuestros días.

\section{Los primeros estudios científicos en el impulso del conservacionismo}

Con el desarrollo industrial y urbano de finales del siglo XIX, se inició una fuerte discusión en el mundo académico internacional que giraba en torno a la recuperación de los valores naturales; esta nueva corriente de pensamiento se denominaría conservacionismo. Pérez-Cebada (2003) destaca dos corrientes predominantes y opuestas, que incluyen un amplio espectro sobre el conjunto de valores éticos y de enfoque en el que debe realizarse la conservación de la naturaleza. El primer enfoque es el conservacionista, este se caracteriza por abierto y optimista sobre las posibilidades que tiene la naturaleza. Además, se fundamenta en el uso de los métodos de análisis científico de origen racionalista y de alta rigurosidad, el uso de la naturaleza es más utilitario. El segundo enfoque es el preservacionista, que le rinde culto a la naturaleza, además asigna valores recreativos y sagrados al bosque. Se dice que es el antecesor de la corriente de pensamiento ecocéntrica.

Debido a las influencias de pensamiento es el primero, es decir, el enfoque conservacionista el que posteriormente empezó a predominar en el consciente de la sociedad costarricense. En contraposición al conocimiento tradicional mantenido por los pueblos indígenas, con claros principios ecocentristas.

Al respecto Goebel, A. (2005-06, p. 4), señala que

La influencia del conocimiento científico, los intereses político - económicos y los condicionamientos estructurales del Estado, presentes en el desarrollo de políticas orientadas a la protección de los recursos naturales, son, asimismo, elementos que pueden contribuir a dimensionar con mayor certeza el origen y 
evolución de las ideas conservacionistas que culminarían con la creación e institucionalización de los Parques Nacionales en Costa Rica.

La apertura del mercado europeo sustentado principalmente en la producción de café y posterior a la Campaña Nacional de 1856-1857, favoreció la llegada de científicos extranjeros que viajaban a explorar Costa Rica para estudiar su geografía y sus recursos biológicos, entre ellos el destacado geógrafo y botánico suizo Henri François Pittier. Personaje que haría grandes contribuciones científicas y a la conservación costarricense. Otros notables científicos fueron el botánico danés Anders Sandre Oerted, el naturalista británico William More Gabb y el ornitólogo lituano Joseph Warscewicz (Evans, 1997). La educación formal era un lujo de unos pocos en ese periodo, por lo que este nuevo flujo de conocimientos fomentó un abordaje más científico de los recursos naturales.

En el año 1886 el Ministro de Educación, don Mauro Fernández, propone una reforma educativa que afectaría la vida académica del país; se cierra la Universidad de Santo Tomás y únicamente funcionaría la Escuela de Derecho. En su lugar se da impulso a la educación secundaria con la apertura de colegios. La reforma proponía sustituir la enseñanza científica por una formación más técnica. Para compensar la carencia de la educación universitaria, la reforma promovió la llegada al país de importantes científicos que reforzarían los nuevos programas de estudio de secundaria. Según Carvajal, (2009), entre ellos los doctores Henry Pittier Dormond, Paul Biolley, Gustave Michaud, Jean Rudin y Adolphe Tonduz, provenientes de Suiza. Su misión sería reestructurar los programas educativos y dar impulso a las ciencias en la secundaria. Se impartieron las cátedras de Ciencias Naturales, Física, Química, Geografía y Agricultura. Además, fue un periodo de gran auge científico, se inauguró el Museo Nacional, inspirado en el Instituto Smithsoniano de Washington, que permitió organizar colecciones botánicas, zoológicas, geológicas y arqueológicas, atrayendo a la comunidad internacional.

El Dr. Henrry Pittier fundó en 1887 el Observatorio Meteorológico, conocido desde el 1888 como el Instituto Meteorológico Nacional. También, fundó el Instituto Físico Geográfico Nacional (IFG), una institución científica de gran incidencia en el desenvolvimiento científico del país. Según Goebel (2006-07, p. 55), se "constituyó como una entidad multifuncional, que concentró gran parte de la actividad científica en aspectos fundamentales para el Estado, tales como la exploración geográfica, el mapeo del territorio, las ob- 
servaciones meteorológicas, y las actividades botánicas”. Entidad que permitió recolectar datos para renovar la cartografía nacional, estudiar los recursos naturales e incentivar la colonización de amplias zonas e integrarse a la economía nacional. Sin embargo, en 1904 el Gobierno de Costa Rica decide incorporar al IFG bajo el auspicio del Museo Nacional, situación que motiva al Dr. Pittier a abandonar al país. Durante su estancia en el país dejó un legado incalculable en el desarrollo científico, por lo que muchos consideran como la era dorada de la historia natural de Costa Rica.

Al jugar un papel de mediación entre la comunidad científica y político, el Dr. Pittier promovió las primeras acciones concretas de conservación. Destacan la promulgación de decretos (Ley) en 1884 para la protección de los bosques nacionales, cauces fluviales y manantiales, así como en 1888 en el decreto (Ley) LXV que se declara la zona inalienable de las montañas de Heredia y Alajuela, con el propósito de proteger las fuentes de agua que abastecen a esas ciudades. El establecimiento de estas zonas de protección ocurrió por la denuncia y la evidencia científica, que indicaba la degradación de los recursos por la tala excesiva de árboles. Otros sucesos estaban relacionados con la degradación de los cauces de los ríos para la extracción de materiales ligados a la construcción del Ferrocarril al Atlántico (Boza, 2015; Evans, 1997). En esa línea también se reafirmó la protección de baldíos y las áreas boscosas próximas a estas, ordenando la limpieza de malezas para evitar la quema de cultivos y bosques. De esta manera, varios de los primeros esfuerzos de conservación en Costa Rica estuvieron vinculados a la protección de las actividades económicas.

La calidad de las colecciones botánicas y la gran variedad de publicaciones científicas orientadas a la descripción y catalogación de especies de flora y fauna influyó en atraer la atención de la comunidad internacional. Para el año 1914, Costa Rica se convirtió en el centro de investigación científico más importante en el trópico americano.

Evans (1997) identifica tres primeras etapas de los aportes científicos en la conservación de Costa Rica: 1) las exploraciones de comienzos del siglo XIX (destacan: Carl Hoffman, Moritz Wagner, Karl Scherzer y Helmuth Polokowski), 2) entre los siglos XIX y XX (destacan: Henri Pittier, C.H. Lankester, J.F. Tristán, Paul Biolley y José Zeledón) y 3) posteriores a la depresión económica (destacan: Alexander Skutch, Clodomiro Picado Twight, José Orozco, Rafael Chavarría, Rafael Lucas Rodríguez Caballero, Archie F. Carr, entre otros) en las que la investigación científica tendió a su declive con respecto a los 
anteriores períodos. En estas tres etapas se dieron hallazgos muy valiosos para entender la diversidad biológica del país y que dejaron una marca en la historia de la conservación.

\section{La degradación ambiental como resultado de la ocupación del territorio}

En la mitad del siglo XX, el ritmo de deforestación en Costa Rica se incrementó de manera alarmante. (Figura 1). Cada vez más los bosques se convirtieron en tierras para el cultivo, plantaciones y pastos asociados a la actividad ganadera. Según Montoya (2004, p. 2) "El reformismo agrario de los últimos años del siglo XIX y principios del XX (1880-1940) estuvo fuertemente determinado por la situación socioeconómica prevaleciente en el país en este período, caracterizado por la expansión cafetalera, las leyes emitidas en ese momento y las consecuencias de su aplicación”.

La intensidad y extensión con la que se explotaron los recursos naturales llevaron a la disminución de la cobertura boscosa del país a proporciones nunca vistas. Boza (2015) estima que entre los años 1940-1950, la cobertura boscosa de Costa Rica cubría el 70\% del territorio, para el 1986 solo quedaba un 21\%. La alta disponibilidad del recurso supuso una explotación casi irracional del mismo. El factor más importante asociado a la tasa de deforestación durante ese período se le atribuye a la actividad agropecuaria (Sierra et al, 2016).

\section{La segunda gran expansión agrícola de Costa Rica}

En 1939, el Estado establece la Ley No. 13 general sobre Terrenos Baldíos con el objetivo de que los campesinos pudieran ocupar lotes baldíos o fincas sin declaración de propiedad particular, y así, regular los conflictos de tenencia de la tierra. Hacia la década de los cincuenta, el país consideró prioritario dirigir la formalización de los terrenos baldíos hacia el fomento del agro.

Según Montoya (2004), sus acuerdos giraron en torno a dos asuntos principales: el denuncio y propiedad de los baldíos y el arrendamiento de tierras nacionales, pero sin alterar el panorama de tenencia de la tierra que se tenía hasta el momento. Según este mismo autor, en 1941 "se dictó una Ley de Informaciones Posesorias, que permitió a quienes probaron haber poseído y explotado tierras sin título legítimo antes de la emisión de la ley de terrenos baldíos de 1939, la adquisición de terrenos entre 100 y 300 hectáreas (caso de 
las fincas ganaderas)” (P.4). Al igual que en períodos anteriores la estructura de la propiedad se mantuvo inalterada.

La primera Asamblea Legislativa (1949-1953), impulsó el proyecto de Ley Agraria, el cual tenía por objetivo mejorar gradualmente las condiciones de las personas que vivían en el campo, mediante el fortalecimiento económico de la familia campesina y la equitativa distribución de las tierras. A partir de 1960 se impulsan una serie de medidas para detener los movimientos emancipadores como la Revolución Cubana. El Plan de la Alianza para el Progreso promovido por Estados Unidos, condicionando el ayuda económico a los países que ejercieran cambios en su estructura agraria y fiscal.

Paralelamente, en 1959, se establece la Ley de Fomento Económico No.2466, en la que se crea el Departamento de Crédito Rural, Tierras y Colonias dentro de la estructura orgánica del Banco Nacional de Costa Rica con la finalidad de hallar soluciones a los problemas del agro. Sin embargo, dicha Ley, en su artículo 48 establece que "La Junta Directiva del Banco Nacional no aprobará ningún plan de adjudicación de tierras a particulares, no otorgará títulos...hasta que la Asamblea Legislativa no emita una Ley General de Tierras y Colonias".

Motivados por ese vació legal, y por la urgencia de implementar programas de reforma agraria en el país, se presenta el proyecto de ley denominado Tierras y Colonización ante la Asamblea Legislativa. Es en la administración del presidente Francisco J. Orlich Bolmarcich (1962-66), bajo la Ley No.3042 que se crea el Instituto de Tierras y Colonización (ITCO) Instituto de Tierras y Colonización (ITCO), el 14 de octubre de 1961, como una institución autónoma, con el fin de implementar el crédito agrícola, la asistencia técnica, la comercialización y distribución de productos (Rodríguez \& Rodríguez, 2007). Es en este período en el cual el Estado tienen un rol fundamental procurar una mejor redistribución de la tierra y a apoyar la modernización de la agricultura.

Según Kay (2005, p. 3), esta modernización “proponía que los países del Tercer Mundo deberían seguir la misma senda que los países capitalistas desarrollados. También contemplaba la penetración económica, social y cultural de los países industrializados del Norte moderno en los países agrarios y rurales del sur tradicional como un fenómeno que favorecía la modernización”. Los cual conllevó a difundir conocimiento, capacidades, tecnología, organizaciones, instituciones, en el campo rural. 
La Revolución Verde, surge en este contexto, como la manera en que los países darían el salto hacia la modernización. Según Castillo. (2008, p. 14):

La Revolución Verde consistía en la utilización de insumos químicos y semillas mejoradas para aumentar la producción de alimentos. Resultados como estos alentaron a los gobiernos de varios países en desarrollo a invertir en investigación y en infraestructura, de tal forma que se facilitara la adopción de las nuevas tecnologías que venían aparejadas a las Revolución Verde. Esto contribuiría, según a beneficiar amplios sectores de producción campesina.

Figura 1. Evolución de la cobertura forestal en Costa Rica y creación de la institucionalidad del Estado Costarricense. Elaboración propia con datos de Fundación Neotrópica y ECG-UNA.
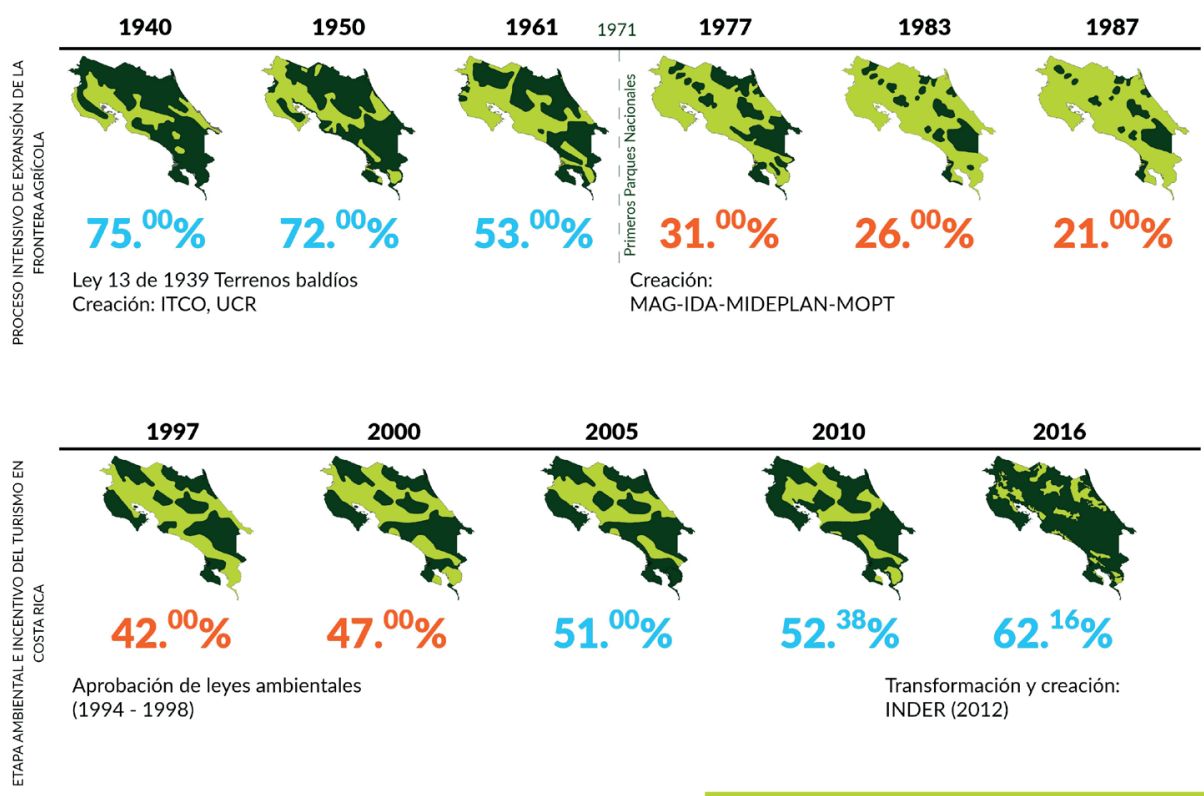

Durante el periodo 1962-66, el Estado promovió la formación de colonias, fomentó la expansión bananera y la formación de empresas agropecuarias, 
que llevó al deterioro de importantes y diversos ecosistemas del país. Además, como política estatal se vigiló que no se realizaran prácticas que atentaran contra la producción agrícola y la crianza de animales. Una medida paliativa para bajar el tono del discurso extractivista implementado por el gobierno fue la creación de las reservas forestales de La Reserva Nacional Absoluta Cabo Blanco en la provincia de Puntarenas en 1963, por el sueco Olof Wessberg y su esposa, la danesa Karen Morgenssen, y Reserva Forestal Río Macho creada en el año 1964; antes del establecimiento del Sistema de Parques Nacionales de Costa Rica (1970).

De acuerdo a los datos recopilados por González (2017) de los Censos Agropecuarios realizados por el Instituto Nacional de Censos (INEC), es posible evidenciar el cambio en los usos del suelo en el país. Es así, como de 1905 a 1973 el fomento al agro incrementó las zonas agrícolas en 703332.45 hectáreas. Esa tendencia de incremento sostenido se mantiene posteriormente en los periodos de 1955 a 1963, lo cual coincide con la Ley de Fomento Económico; y en periodo de 1963 a 1973 motivado por la creación de Instituto de Tierras y Colonización (ITCO) y los créditos otorgados por parte del Banco Nacional para incentivar el crecimiento del sector agropecuario.

De 1973 a 1984 el suelo agrícola desacelera su crecimiento, como respuesta a la reorientación de la política del agro costarricense, en la que se traspasó la concentración de la tierra en poder de las grandes explotaciones o latifundios, a los minifundios con especial atención a la constitución de fincas familiares, aunque con ellos aumentó la intensidad del uso del factor tierra. En 1982, mediante la Ley No. 6735 se transforma el ITCO en el Instituto de Desarrollo Agrario (IDA), con el fin de enfocarse en elevar la productividad agrícola, mejorar la infraestructura agropecuaria y administrar las tierras productivas del país (González, 2017). Dos años después de la transformación del IDA, el número de hectáreas destinas a la producción agrícola empieza a disminuir, no así la cantidad de fincas registradas para tal fin. Esto supone un proceso de parcelamiento más intenso, a partir de fincas madres, y relacionado con el otorgamiento de terrenos a pequeños productores que orientaron su producción al nuevo modelo incentivado por el Ministerio de Agricultura y Ganadería (MAG).

El proceso de colonización fomentó la construcción de infraestructura vial por el Ministerio de Obras Públicas y Transportes (MOPT), para que comunicase los lugares de producción con los mercados de consumo. De gran importancia fue la construcción de la Carretera Interamericana obra que se 
inició en el año 1942 y quedó transitable para el año 1963; conectó al país de norte a sur (desde Peñas Blancas en la frontera norte con Nicaragua hasta Paso Canoas en la frontera sur con Panamá). La ocupación y expansión agrícola del centro del país hacia las áreas periféricas motivó la cantidad y densidad de vías en el país, las cuales se pueden asociar al crecimiento de las áreas destinadas al agro. Paralelamente se da una fragmentación de las áreas naturales con el incremento de la ocupación y cambios de uso de la tierra. En el mapa 1 se relaciona la longitud de la red hídrica con la red vial, lo que evidencia una tendencia a la construcción de vías de acceso, donde se muestra que por cada kilometro de cauces existen 798 metros de vías.

Mapa 1. Longitud de red hídrica / Longitud de vialidad. Elaboración propia.
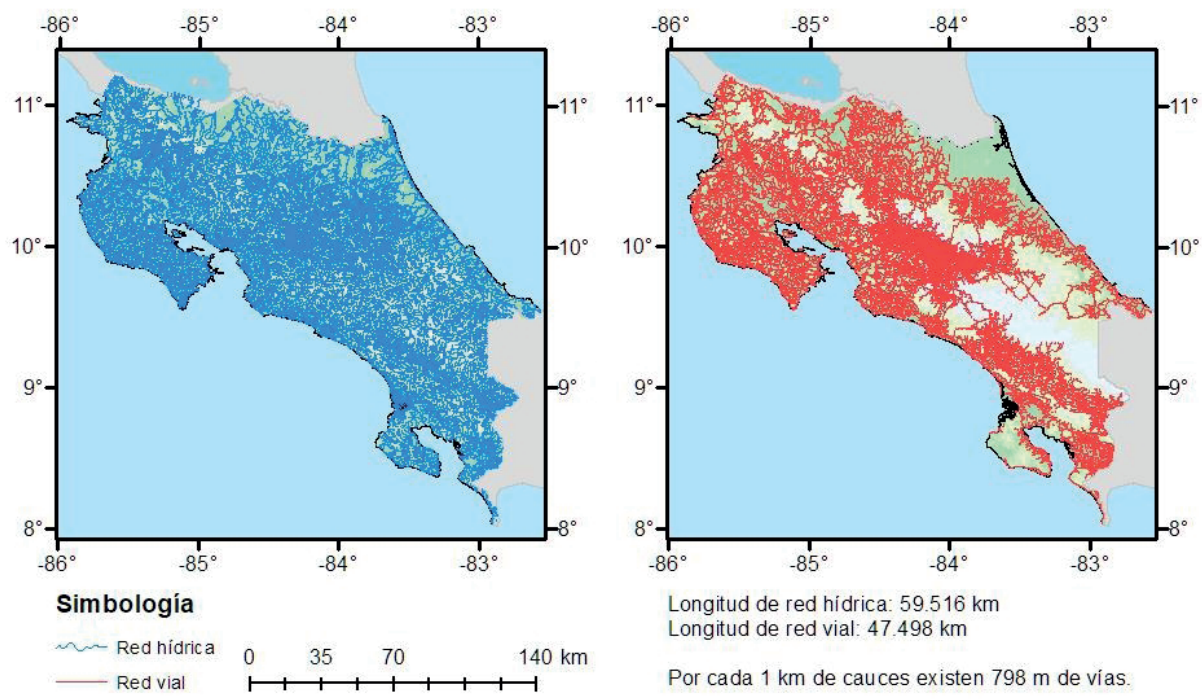

Incidentemente la Ley General de Caminos Públicos y la Ley Nacional de Fraccionamiento y Urbanizaciones, señalan que cuando se constituye una vía de acceso público, se abre la posibilidad de urbanización frente a la vía pública. Por lo que las instituciones y organizaciones que brindan los servicios básicos deben garantizarlos a los habitantes si estos lo solicitan. Es así como el Instituto Costarricense de Electricidad (ICE) y el Instituto de Acueductos y 
Alcantarillados (AyA) consecuentemente formaron parte de los procesos de disminución de los bosques costarricenses.

El crecimiento urbano, también está asociado al desarrollo agropecuario, ya que muchas ciudades periféricas tenían una funcionalidad de apoyo a la actividad agrícola. Además, es importante señalar que los procesos de urbanización son más probables que ocurran en aquellos terrenos que una vez fueron ocupados por el agro. En la tabla 1, se aprecia el comportamiento de las hectáreas dedicadas a pastos, con un incremento contante en los períodos intercensales indicados.

En el 2012 con la transformación del IDA al Instituto Nacional de Desarrollo Rural (INDER), el sistema agro pastoril en Costa Rica empieza a cambiar la estructura de la ocupación y utilización de la tierra. Se busca instaurar un modelo de ocupación más integral, que dé más valor a un tipo de producción sostenible que contribuyan a la preservación de la biodiversidad y a la generación de servicios ambientales o ecosistémicos se ha transformado, y el cual tiende a rescatar el modelo de uso que daba el campesinado en la Colonia. De esta manera, se incentiva en las fincas, la conservación áreas de bosque en conjunto con las zonas de pastoreo y agricultura. Este modelo, es aún muy incipiente para determinar si su implementación ha traído beneficios generales a los esfuerzos de conservación en el país. Aunque, es una manera lógica de convertir lo que fue una de las principales causas de la degradación de la cobertura boscosa de Costa Rica en una alternativa de conservación. 
Tabla 1. Pastos y su clasificación en los censos Agropecuarios

\begin{tabular}{|c|c|c|}
\hline Censo & Terminología & Área en hectáreas \\
\hline 1905 & Potrero artificial y natural & 211171.32 \\
\hline 1955 & $\begin{array}{c}\text { Pastos permanentes: potrero (pastos bajos) y pastos } \\
\text { naturales, repastos (pastos altos cultivados) } \\
\text { Pastos de Corte }\end{array}$ & \multirow{2}{*}{1014503.1} \\
\hline 1963 & $\begin{array}{c}\text { Potrero y pastos bajos (naturales), repastos (cultivados) y } \\
\text { pastos altos Pastos de corte }\end{array}$ & 1242675.77 \\
\hline 1973 & $\begin{array}{c}\text { Potreros (pastos naturales) Pastos cultivados o mejorados } \\
\text { para no corte Pastos cultivados o mejorados para corte }\end{array}$ & 1558053.1 \\
\hline 1984 & Pastos mejorados o cultivados para pastoreo \\
& Pastos naturales Pastos para corte & 1651560.5 \\
\hline
\end{tabular}

Fuente: Adaptado de González (2017). 


\section{Mapa 2. Costa Rica: Densidad de fincas ganaderas de carne o doble propósito en $\mathrm{km}^{2}$ en el 2018. Elaboración propia.}

Costa Rica: Densidad de fincas ganaderas de carne o doble propósito en $\mathrm{km}^{2}$

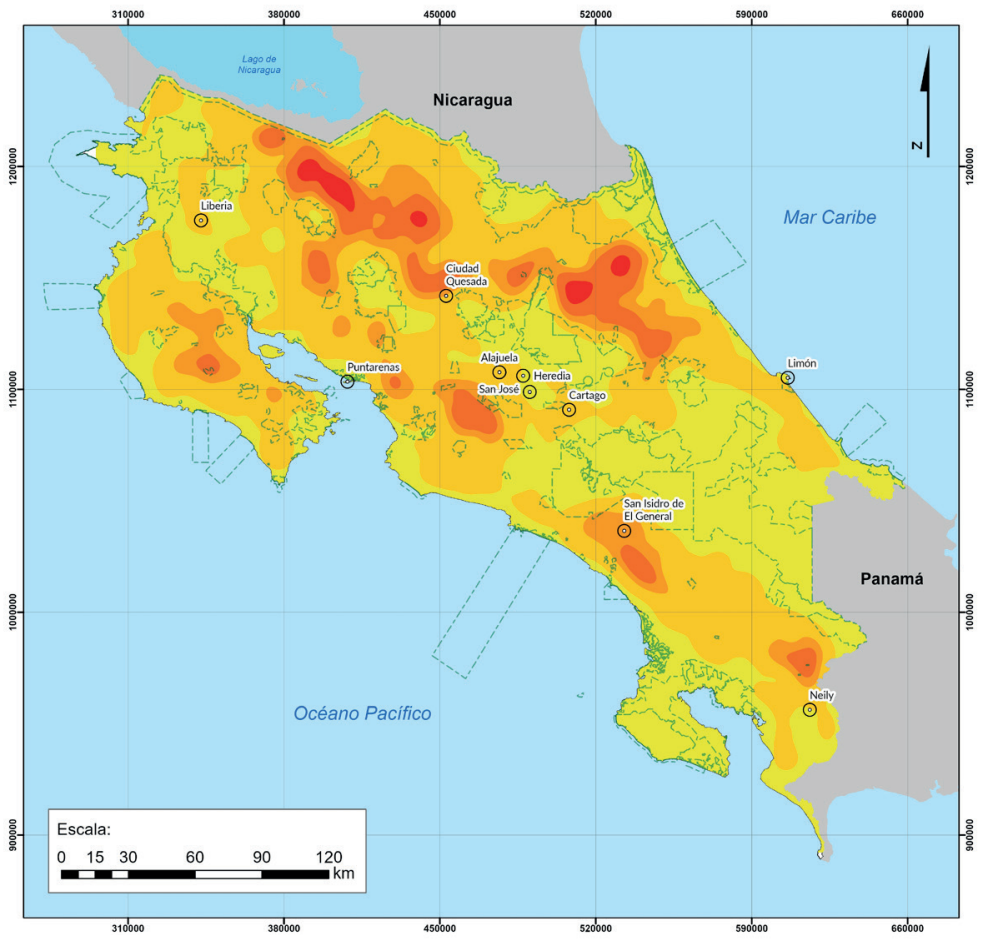

\section{Simbología}

$\odot$ Ciudades

Áreas silvestres protegidas

Análisis de densidad $\left(\mathrm{km}^{2}\right)$

Intervalo

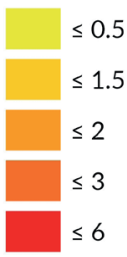

\section{Fuente Cartográfica:}

SENASA. (2018). Fincas ganaderas. Ubicación de Asociados CORFOGA. San José.

IGN. (2007). División Político Administrativa. San José: CENIGA.

IGN. (2007). Poblados. San José: CENIGA.

\section{Fuente Fotogramétrica:}

Programa de Regularización de Catastro y Registro Gobierno de Costa Rica. (2005). Ortofotos y restitución fotogramétrica. Escala 1: 5000 . San José.

\section{Escuela de Ciencias Geográficas: \\ SIG y Diseño Gráfico:}

Geóg. Omar E. Barrantes Sotela

Mayo, 2019.

Análisis de densidad de fincas ganaderas productoras de carne mediante o de doble propósito. Estimación de densidad del kernel mediante: Quartic (biweight). Radio de búsqueda establecido en $\mathrm{km}^{2}$. Proyección métrica CRTM05. 
Mapa 3. Costa Rica: Densidad de cabezas de ganado de carne o doble propósito en $\mathrm{km}^{2}$ en el 2017. Elaboración propia.

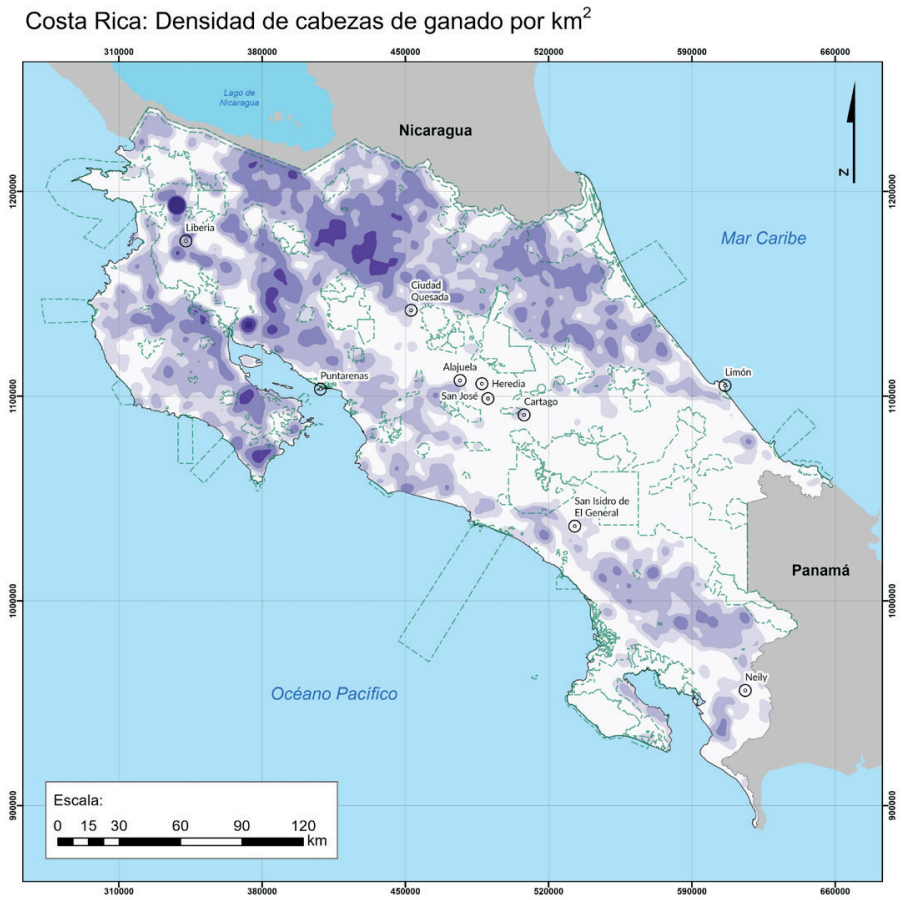

\section{Simbología}

() Ciudades

Áreas silvestres protegidas

Densidad (cabezas de ganado por $\mathrm{km}^{2}$ )

Intervalo

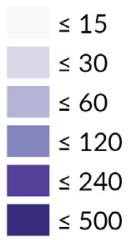

Fuente Cartográfica:

SENASA. (2018). Fincas ganaderas. Ubicación de Asociados CORFOGA. San José

IGN. (2007). División Político Administrativa. San José: CENIGA.

IGN. (2007). Poblados. San José: CENIGA

\section{Fuente Fotogramétrica:}

Programa de Regularización de Catastro y Registro Gobierno de Costa Rica. (2005). Ortofotos y restitución fotogramétrica. Escala 1: 5000 . San José.

Escuela de Ciencias Geográficas:

SIG y Diseño Gráfico:

Geóg. Omar E. Barrantes Sotela

Mayo, 2019

Análisis de densidad de población total de ganado con fines de carne o doble propósito por $\mathrm{km}^{2}$. Estimación de densidad del kernel: Quartic (biweight). Radio de búsqueda establecido en $\mathrm{km}^{2}$. Proyección métrica CRTM05. 


\section{El impulso a la creación de las Áreas Silvestres Protegidas (ASP) de Costa Rica}

Costa Rica era el país con mayor pérdida de bosque en Centroamérica, muchos de sus terrenos mostraban serios signos de deterioro ambiental generalizado como la pérdida y erosión de suelo, degradación de las cuencas hidrográficas, pérdida de biodiversidad y de ecosistemas (Barrantes, 2000; Barrantes y Castro, 1999). Con estos antecedentes ¿Por qué Costa Rica es ahora conocida por ser un ejemplo de conservación en el mundo? ¿Por qué el turismo ha logrado aprovechar el patrimonio natural y la gran biodiversidad que este representa?

Buena parte de la respuesta se debe a la creación de un modelo conservacionista basado en sus áreas silvestres protegidas (ASP). Según Barrantes (2000), Boza (1993 y 2015) y Evans (1997), ante la crisis ambiental generada por la rápida tasa de deforestación se dio una fuerte reacción que propició la protección de las manchas más representativas de biodiversidad. La Ley (No. 4465) Forestal de 1969, fue la que inicialmente definió las principales clases de áreas protegidas de Costa Rica. El artículo 35 de esta ley, define las categorías del patrimonio forestal del Estado en: a) Reservas forestales, b) Zonas protectoras, c) Parques nacionales, d) Refugios nacionales de vida silvestre y e) Reservas biológicas. Al estudiar la terminología usada en las definiciones de las primeras categorías de conservación, se refleja una perspectiva de conservación utilitaria con una gran influencia del modelo de conservación y manejo de vida silvestre de la escuela estadounidense. Por ejemplo, se indica que la principal función del bosque es la de reserva para la producción de madera.

Según Wallace (1992), Evans (1997) y Boza (2015), la importancia de las áreas silvestres protegidas es multidimensional. En un sentido amplio, el objetivo principal de las ASP es preservar el patrimonio natural y cultural de Costa Rica. Esa no es una tarea tan sencilla en un país que dispone de una diversidad tan amplia de ecosistemas y zonas de vida, en la que se sobreponen intereses para el desarrollo de los usos agrícolas y económicos de la tierra. Es importante destacar que el Servicio de Parques Nacionales fue creado en 1977 por la Ley 6084 como una dirección general o departamento dentro del Ministerio de Agricultura y Ganadería, lo que suponía una contradicción, ya que era el ministerio que promovía la expansión agrícola en Costa Rica.

El Servicio de Parques Nacionales, empezó la identificación de las zonas geográficas con ecosistemas representativos e inició las acciones para su protección. Para lograr los objetivos de conservación se establecieron cinco propósitos 
específicos a conservar: los históricos y arqueológicos, los entornos montañosos y volcánicos, el bosque tropical seco, el bosque tropical húmedo y los ecosistemas subterráneos y submarinos. Según Carr (1982) y Janzen (1986), el valor científico de este sistema de preservación es probablemente inconmensurable. Es así como el Parque Nacional Santa Rosa, el Parque Nacional Guanacaste, y el Parque Nacional Palo Verde, son los únicos bosques tropicales secos protegidos del mundo. Mientras que el Parque Nacional Volcán Poás es uno de los pocos volcanes activos que quedan en el mundo con acceso ${ }^{4}$ durante todo el año para estudios científicos.

Del mismo modo, mantener un estado lo más natural posible para las comunidades de plantas y animales tropicales representa una fuente interminable de material educativo y de investigación para todas las edades (Fournier, 1991). El Sistema de Áreas Protegidas, es considerado también como un banco de material genético que tiene potencial científico, médico y económico. Es así como Evans (1997), considera que el valor genético de la protección de especies en el hábitat protegido por los parques y reservas de Costa Rica puede mantener al país en el centro de atención científica durante las próximas décadas.

Como diversos son los entornos naturales en Costa Rica, así han sido los intereses para la creación y conformación de las múltiples Áreas Silvestres Protegidas (ASP). En algunos casos el Estado costarricense fue el propulsor por captar fondos de ayuda financiera del exterior, como es el caso del Parque $\mathrm{Na}$ cional Santa Rosa. En otros, ocurrió por fuertes iniciativas privadas y de grupos organizados; el resultado son distintas formas de manejo administrativo. Tal vez, uno de los ejemplos más significativos es el manejo compartido en el Parque Nacional Cahuita, entre los habitantes de la comunidad y el Ministerio de Ambiente y Energía (MINAE).

4 En este momento en vigilancia y monitoreo por el OVSICORI y la RSN debido a las constantes erupciones volcánicas y la amenaza que significa para los habitantes cercanos. 
Mapa 4. Costa Rica. Parques Nacionales, 1979. (Servicio de Parques Nacionales, MAG)

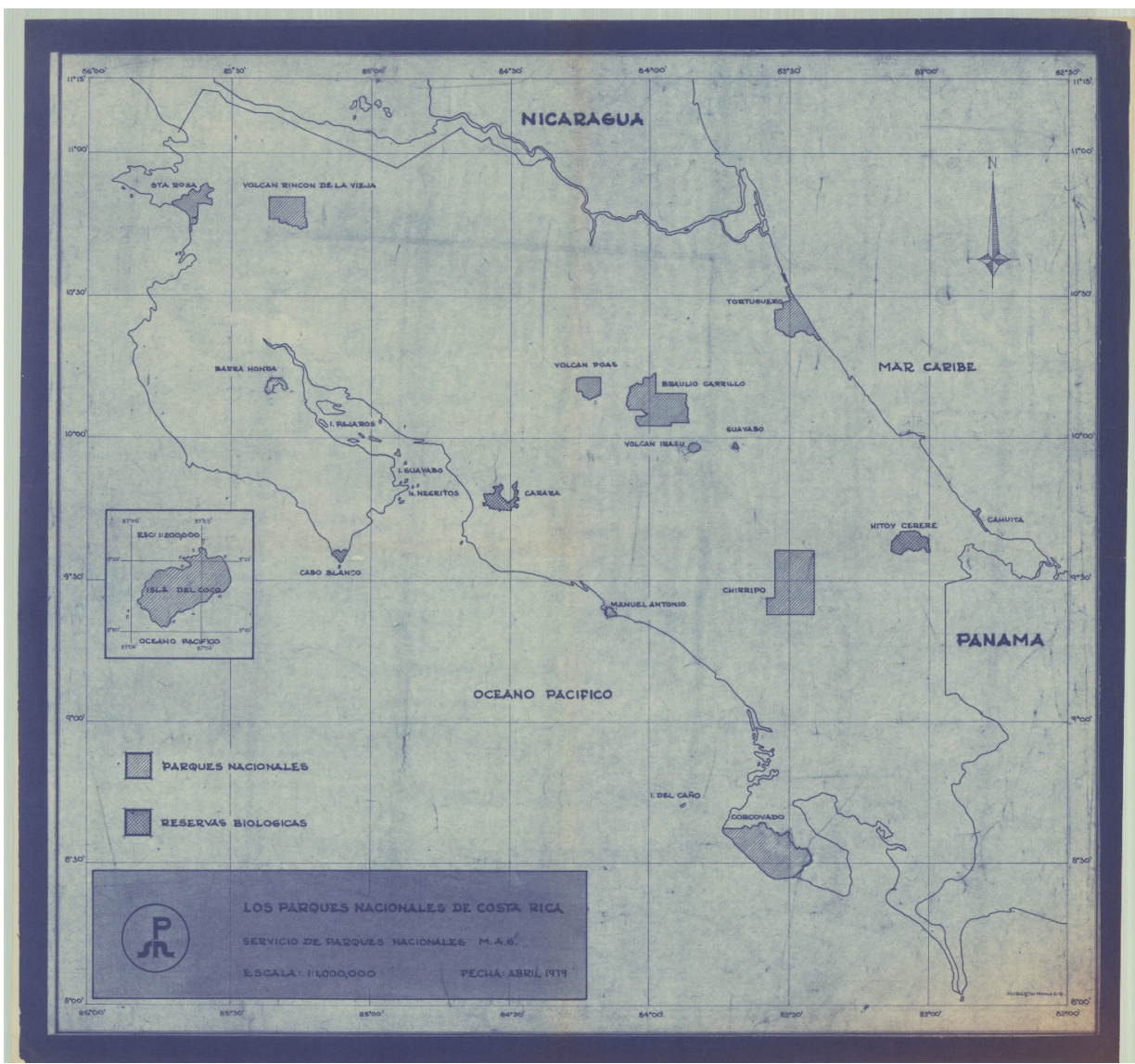

Con la promulgación de la legislación ambiental en la década de los años noventa, se dio un giro muy peculiar en el desarrollo y la gestión de las ASP. De esta manera los procesos de desconcentración y descentralización del Estado, también afectó el desenvolvimiento administrativo de las Áreas de Conservación. Además, la ley 7152 había trasladado el Servicio de Parques Nacional del MAG al MINAE y se conocería posteriormente como el Sistema Nacional de Áreas de Conservación (SINAC). No obstante, si bien el enfoque utilitario de las ASP siempre se mantiene, ahora busca integrar los conceptos teóricos provenientes del Desarrollo Sostenible. En el Mapa 4 se muestra los primeros 
parques nacionales y en el Mapa 5 se muestra la actual distribución de las Áreas Silvestres Protegidas, según categoría, resaltando el hecho de que los Parques Nacionales tanto terrestres como marinos sobresalen como categoría de manejo, en la cual no se realiza actividades económicas, excepto la visitación turística, en una pequeña proporción de su territorio, en algunos parques menos del $2 \%$ son aprovechas para fines recreativos y educativos. En la tabla 2 se muestra la superficie destinada a la conservación según la categoría de manejo.

Mapa 5. Costa Rica. Áreas Silvestres Protegidas, 2019.

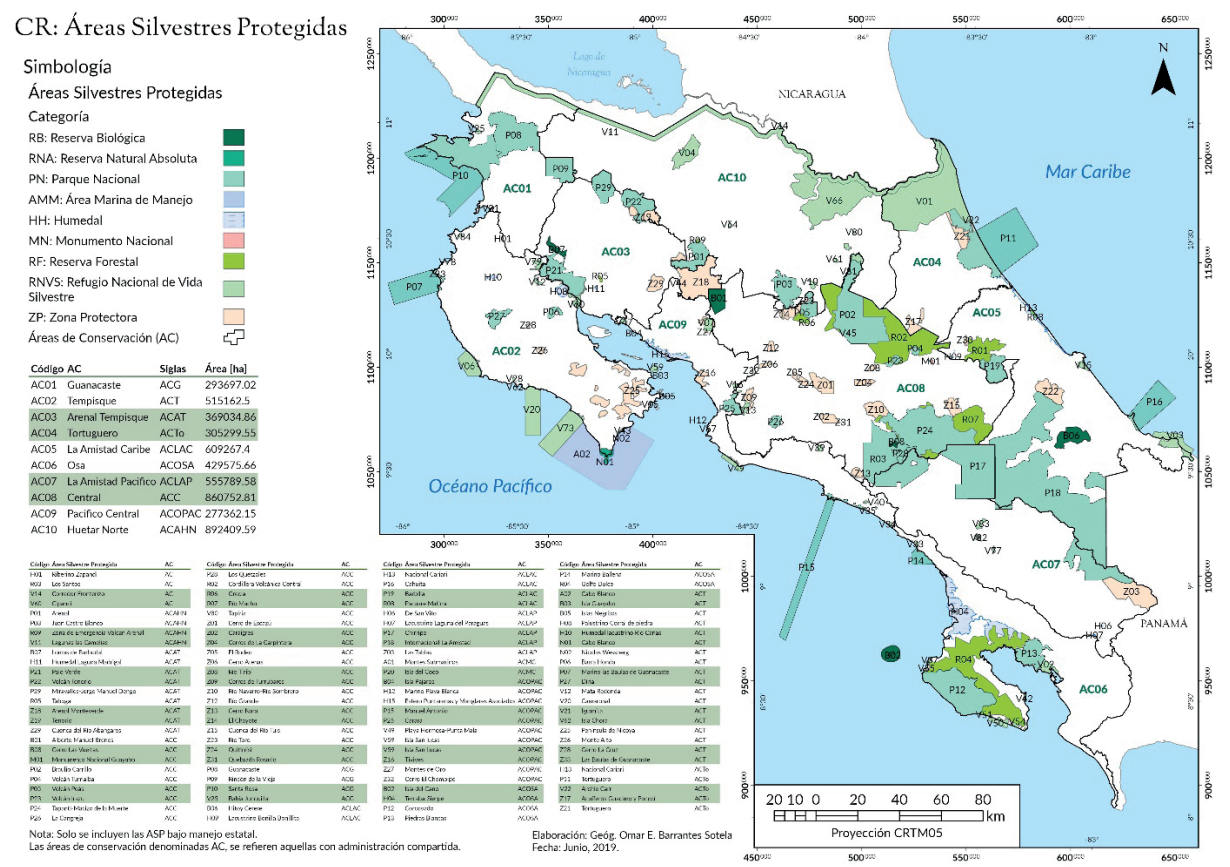


Tabla 2. Superficie destinada a la conservación según categoría de manejo en Costa Rica.

\begin{tabular}{|c|c|c|c|c|}
\hline & & $\begin{array}{c}\text { Área } \\
\text { Terrestre }\end{array}$ & $\begin{array}{l}\text { Área } \\
\text { Marina }\end{array}$ & Islas \\
\hline Categorías de Manejo & $\begin{array}{l}\text { Total, de } \\
\text { ASP }\end{array}$ & $\mathrm{Km}^{2}$ & $\mathrm{Km}^{2}$ & $\mathrm{Km}^{2}$ \\
\hline Parque Nacional & 28 & 6299.18 & 1721.83 & 2045.25 \\
\hline Reserva Natural Absoluta & 2 & 14.11 & 16.88 & 0.17 \\
\hline Monumento Natural & 1 & 2.30 & 0.00 & 0.00 \\
\hline $\begin{array}{l}\text { Refugio Nacional de Vida } \\
\text { Silvestre }\end{array}$ & 35 & 2295.26 & 554.43 & 4.71 \\
\hline Reserva Biológica & 8 & 211.84 & 52.01 & 4.56 \\
\hline Reserva Forestal & 9 & 2159.60 & 0.00 & 0.00 \\
\hline Zona Protectora & 31 & 1557.25 & 0.00 & 0.00 \\
\hline Humedal & 11 & 363.35 & 0.07 & 0.00 \\
\hline Área Marina de Manejo & 2 & 0.00 & 10443.31 & 0.00 \\
\hline Total & 127 & 12902.89 & 12788.53 & 2054.69 \\
\hline
\end{tabular}

Fuente: Adaptado de SINAC (2017).

\section{Las Áreas Silvestres Protegidas (ASP) y los conflictos por la conservación}

La conservación también ha provocado diversos conflictos. Los primeros se dieron por la forma en la que se adquirieron los terrenos destinados para la conservación. En las primeras áreas silvestres medió un despojo institucionalizado de varias poblaciones, y en los que los pagos por expropiación en el mejor de los casos se resolvieron años después. También, fue común los problemas entre los guardaparques y los habitantes de los poblados cercanos, que incluso generaron conflictos transgeneracionales por las discrepancias en el aprovechamiento de los recursos naturales. 
Otro caso especial, es el de los pueblos indígenas que fueron excluidos de los esfuerzos de conservación del Estado, y que en su cosmovisión mantienen preceptos y valores asociados al uso sostenible del medio ambiente. Es importante señalar que el deterioro ambiental también implica la pérdida de conocimientos ancestrales como plantas medicinales, tintes naturales, el uso de técnicas constructivas en edificaciones, así como la pérdida de sistemas autóctonos de producción (Corcuera y Ponce de León, 2004; Pisquiy Pac, 2008). Una nueva dimensión a los conflictos por la conservación se inició con el auge del narcotráfico. En especial en aquellas áreas protegidas con superficies en el mar, o que por sus condiciones de aislamiento favorecen el trasiego de estupefacientes (Sesnie et al., 2017).

\section{El patrimonio territorial y el turismo en el modelo conservacionista}

Según, Albarrán, D. (2016, p. 222), citando a Florido, 2013, señala que "El concepto de Patrimonio Territorial, en su concepción más general, ha sido definido como el entendimiento del espacio geográfico como objeto de interés patrimonial en tanto que resultado de la permanente interacción de hombre y medio y que insta a observar, interpretar y gestionar de manera unitaria el conjunto de componentes que lo conforman, tanto naturales como culturales".

Partiendo de la definición anterior, desde inicios del siglo XX el turismo se ha convertido en una actividad asociada a la conservación de los recursos naturales en Costa Rica. La creación del Instituto Costarricense de Turismo (ICT) en el año 1955 inicia con la modalidad del turismo de playa y naturaleza en el país. Debido a las facilidades de la oferta de transporte y al desarrollo de una red vial más densificada.

Según, Quirós y Sánchez (2014, p. 14),

a diferencia de otras regiones del mundo, América Central y gran parte de México incentivan tardíamente la visitación de turistas a la región. La carencia de grandes complejos turísticos, la limitada infraestructura y la promoción turística centrada especialmente en los recursos de sol y playa que privilegian la visitación de destinos masivos y altamente promocionados, mantuvieron a la región alejada del escenario mundial del turismo.

Sin embargo, ya para la década de los 1980's según Nel y Andreu señalan que "Costa Rica irrumpió en la escena del turismo internacional y apostó por el 
turismo basado en sus atractivos naturales, más concretamente por el ecoturismo, como modelo de desarrollo económico, hace ya más de dieciséis años, posicionándose a nivel mundial como destino ecoturístico de reconocido prestigio y principal destino turístico de Centroamérica” (2008, p. 168).

En esta orientación, Costa Rica ha dirigido su desarrollo económico y social hacia un esquema globalizado y de libre comercio, incursionando en la producción de productos tradicionales y no tradicionales. Lo que coloca al turismo como una alterativa para obtener diversidad de beneficios económicos (Lippmann, 2005 y Northrup, 2012).

Tabla 3. Áreas Silvestres Protegidas con mayor visitación de turistas residentes y no residentes.

\begin{tabular}{cccc}
\hline Área Silvestre & Visitantes residentes & $\begin{array}{c}\text { Visitantes no } \\
\text { residentes }\end{array}$ & Total \\
\hline Manuel Antonio & 67160 & 164625 & 231785 \\
Volcán Poás & 100086 & 95483 & 195569 \\
Volcán Irazú & 75387 & 18170 & 93557 \\
Marino Ballena & 56223 & 20681 & 76904 \\
Arenal & 13392 & 50086 & 63478 \\
Rincón de la Vieja & 8278 & 32414 & 40692 \\
Tortuguero & 9741 & 29774 & 39515 \\
Volcán Tenorio & 21891 & 10994 & 32885 \\
Cahuita & 10000 & 22774 & 32774 \\
Corcovado & 7065 & 22855 & 29920 \\
Santa Rosa & 18624 & 14827 & 24864 \\
Carara & 7764 & & 2591 \\
\hline
\end{tabular}

Fuente: Adaptado de DE-SINAC (2017). 
Una de las formas de aprovechar la diversidad biológica del país fue mediante la construcción de un modelo turístico basado en las directrices internacionales para el desarrollo sostenible. Por sus riquezas naturales el país ha logrado posicionarse a nivel mundial como un destino ecológico y sustentable. De acuerdo al Plan Nacional de Desarrollo Turístico de Costa Rica 2017-21 del ICT (2017), las ASP constituyen el grupo de atractivos turísticos de mayor importancia en el país. De igual forma para la mayoría de los viajeros las ASP son el principal motivo para viajar al país por su afinidad con temas ambientales y de sostenibilidad ambiental. De manera generalizada, las ASP cuentan con facilidades y condiciones para el consumo de "productos turísticos específicos" como lo son la observación de aves, avistamiento de tortugas, caminatas de alta intensidad, vulcanismo y ecosistemas específicos, entre otros.

A pesar de que el Sistema de Áreas de Conservación en su conjunto es el que contribuye con la imagen ecologista del país, la actividad turística se concentra en 12 ASP (Tabla 3). Por lo que no es casualidad que desde el año 2011, se invierte en mejoras de la infraestructura turística de las ASP: Rincón de la Vieja, Caño Negro, Arenal, Tortuguero, Cahuita, Corcovado, Manuel Antonio, Poás y el Irazú. Mediante el proyecto Banco Interamericano de Desarrollo (BID), Parques-SINAC con el apoyo del Instituto Costarricense de Turismo (ICT).

La imagen turística de Costa Rica está fuertemente ligada a sus prácticas ecológicas; por lo que la mayor amenaza para este sector es la degradación de sus recursos naturales. Sin embargo, el país invierte sólo una pequeña parte de sus recursos para la resolución de los problemas ambientales causados por el desarrollo urbano descontrolado, la explotación maderera, la cacería, y la tenencia privada dentro de las ASP.

Tal y como relata Evans (1997) y Boza (1993 y 2015), siempre se mantuvo una posición de crear un programa de parques que se mantuviese a la vanguardia del fenómeno de la recreación del turismo al aire libre a fin de preservar y proteger la dotación de recursos naturales del país desde un enfoque del desarrollo sostenible. Es así como el éxito del Sistema Nacional de Áreas Protegidas es importante a nivel internacional, debido a la espectacular diversidad ecológica de Costa Rica, que puede ser disfrutada por los turistas. Por tanto, garantizar fondos provenientes de la actividad turística para ser invertidos en el programa era algo deseado para fortalecer las acciones de conservación. 
A pesar de las prácticas sostenibles plasmadas en programas y políticas, en mayo del 2003 Ministerio de Ambiente y Energía (MINAE), anunció que debía realizar un plan para cerrar los Parques Nacionales por falta de presupuesto para su mantenimiento y administración (Pratt, 2002). Muestra de ello es que de un total de 161 ASP, del año 2011 a la fecha sólo se ha mejorado la infraestructura de las 12 ASP más visitas por los turistas (Lara, 2017). De manera contradictoria el sector asociado a la actividad turística es uno de los mayores beneficiados de los recursos naturales, es también el sector que menos invierte en su protección y mejoramiento. Además, solo en dos casos particulares, el SINAC cuenta con convenios y acuerdos entre entes privados, cifra relativamente pequeña al considerar que existen más de 90 convenios firmados entre esa institución e instituciones del estado costarricense, Organizaciones No Gubernamentales y Fundaciones.

Otro aspecto interesante, es lo estipulado en el Decreto Ejecutivo No. 37669-RE-COMEX-TUR y su reglamento en el que se establece la marca país como un esfuerzo para promocionar la autenticidad de los costarricenses y que define al mismo como "país verde", con el propósito de mejorar el posicionamiento en la realización de negocios para atraer turistas, inversionistas y compradores extranjeros al país. Es una apropiación estatal del "sentir costarricense" y que en el artículo 4 del reglamento asocia un mensaje de que "Costa Rica concentra, posee, potencia y cuida aquello que es clave para el futuro: biodiversidad, belleza natural única, calidad, talento humano especializado, innovación, una ubicación privilegiada, diversidad de pueblos indígenas, valor agregado en sus productos y profesionales".

No obstante, las empresas o personas jurídicas que opten para el uso de la Marca País deben cumplir con una serie de requisitos y que son evaluados por criterios de evaluación comerciales y empresariales. Es decir, se convierte en una certificación estatal que vende una imagen al visitante que no es consecuente con el abanico de opciones turísticas ofrecidas. Ya que, además de un turismo de naturaleza se ofrece turismo de casinos y golf siempre verde, siendo este último una amenaza para los ecosistemas sensibles. Lo anterior, se ha evidenciado en el conflicto dado entre las comunidades y los megaproyectos turísticos en el Golfo de Papagayo (Pratt, 2002). Aún más preocupante, el país se enfrenta a una depreciación de su imagen conservacionista, ya que su política ecológica no corresponde con el manejo que se les da a los desechos sólidos, aguas negras, servicio de alcantarillado, implementación de energías limpias y la regulación 
urbana. Su patrimonio territorial más preciado, los recursos naturales, muestra signos de deterioro, sin que las políticas creadas para potenciar los mismos, logren frenar este proceso, no al ritmo deseado.

\section{Conclusiones}

La colonización agrícola del territorio conllevó una gran alteración ambiental de los territorios, especialmente la deforestación de amplias zonas que se abrieron para dar paso a usos del suelo agrícola y residencial. Lo que implicó para el país pasar de una cobertura boscosa a una deforestación sin presentes en la segunda mitad del siglo pasado. La política pública por un lado motivó la ocupación de estos espacios, pero que igualmente reacciona ante la degradación ambiental; consolidándose a finales de siglo pasado un sistema de áreas de conservación, que constituyen la vanguardia en términos de conservación y del turismo.

Los esfuerzos de conservación en Costa Rica responden a múltiples intereses y están en función a la atención de una crisis. Las motivaciones de respuesta pueden ser muy variadas como la conservación biológica, cultural e histórica. Además, en la mayoría de los casos los lineamientos estatales concernientes a la conservación se basan en la viabilidad económica y política. Mientras que los criterios de manejo y conservación de la biodiversidad mantienen un enfoque utilitario a través del tiempo.

Uno de los aspectos que debe reforzarse en las ASP, es el fomento del vínculo con las comunidades locales. De manera especial en aquellos casos en el que los conflictos ambientales continúan o no fueron bien atendidas. Además, las políticas del Estado costarricense no están articuladas a las acciones de conservación, en especial aquellas orientadas a promover actividades agropecuarias y turísticas con un alto impacto al medio ambiente.

El turismo que se convierte desde los años 80 's en una importante fuente de ingresos económicos a través de la generación de divisas, podría poner en riesgo la fuente de recursos en los cuales basa su éxito. Sigue siendo necesario una visión a largo plazo, y que la sustentabilidad de las áreas naturales protegidas se base en una estrategia articulada con el patrimonio territorial que dan sustento al desarrollo del turismo. 


\section{Referencias}

ALBARRÁN, D. "El concepto de Patrimonio Territorial: problemáticas de gestión planificación turística”. In: BLÁZQUEZ, M., et al (eds.). "Turismo y crisis, turismo colaborativo y ecoturismo". XV Coloquio de Geografía del Turismo, el Ocio y la Recreación de la AGE, Mon. Soc. Hist. Nat. Balears, n. 23 pp. 67-78, 2016.

BARRANTES, G. Aplicación de incentivos a la conservación de la biodiversidad en Costa Rica. San José: 2000.

BARRANTES, G., y CASTRO, E. Aportes de la biodiversidad silvestre de las áreas de conservación a la economía nacional. San José: 1999.

BOZA, M. "Society for Conservation Biology Conservation in Action: Past, Present, and Future of the National Park System of Costa Rica". Conservation Biology, n. 7 v. 2, pp. 239-247, 1993.

BOZA, M. Historia de la conservación de la naturaleza en Costa Rica 1752-2012. Cartago: Editorial Tecnológica de Costa Rica, 2015.

CARVAJAL, G. “La institucionalización de la geografía en Costa Rica”. En: A., Sánchez y A., Liberalli (eds.), La geografía en América Latina: visión por paises. Buenos Aires, pp. 43-54, 2009.

CASTILLO, O. Paradigmas y conceptos de desarrollo rural. Pontificia Universidad Javeriana, Bogotá, Colombia, pp. 1-61, 2008.

CARR, A. "Tropical forest conservation and estuarine ecology". Biological Conservation, n. 23, v. 4, pp. 247-259, 1982. https://doi.org/https://doi.org/10.1016/00063207(82)90081-7.

CORCUERA, P., \& PONCE DE LEÓN, L. “Tendencias de los movimientos conservacionistas y el surgimiento de la Eco-Ética”. Sociológica, n. 19, v. 56, pp. 199-211, 2004.

DE-SINAC. Presupuesto 2017. San José, 2017.

EVANS, S. D. The green republic: A conservation history of Costa Rica, 1838-1996. ProQuest Dissertations and Theses. University of Kansas: 1997. 
FOURNIER, L. A. Desarrollo y perspectiva del movimiento conservacionista costarricense. San José: Editorial de la Universidad de Costa Rica, 1991.

GOEBEL, A. “Ciencia, legislación y discurso conservacionista. El 'germen' de los Parques Nacionales en Costa Rica: elementos contextuales y 'matices' analíticos 1833-1955". Diálogos Revista Electrónica de Historia, n. 6, v. 2, pp. 1-39, 2005-06.

GOEBEL, A. “Economía, ciencia, y 'liberalismo’: Condicionamientos económicos de la institucionalidad científica liberal en Costa Rica. Una invitación al análisis. 1887-1910". Diálogos Revista Electrónica de Historia, n. 7, v. 2, pp. 51-94, 2006-07.

GONZÁLEZ, V. Análisis histórico de la estructura agropecuaria de Costa Rica: Primeros Indicios. Costa Rica: Informe Estado de La Nación, 2017.

GRANADOS, C. "El impacto ambiental del café en la historia costarricense". Revista Diálogos, n. 4, v. 2, pp. 120-53, 2004.

HALL, C. El café y el desarrollo histórico-geográfico de Costa Rica. Editorial Costa Rica y Universidad Nacional. San José, Costa Rica, 1976.

HALL, C. Costa Rica: una interpretación geográfica con perspectiva histórica. San José, Costa Rica: Editorial Costa Rica, 1984.

HALL, C. "América Central como región geográfica”. Anuario de Estudios Centroamericanos, n. 11, v. 2, pp. 5-24, 1985.

ICT. Plan Nacional de Desarrollo Turistico de Costa Rica. San José, 2017.

JANZEN, D. H. “The eternal external threat”. In: M. E. Soulé (Ed.), Conservation Biology. Sunderland: Sinauer, 1986, pp. 286-303.

KAY, C. "Los paradigmas del desarrollo rural en América Latina. Institute of Social Studies, La Haya". En: "El mundo rural en la era de la globalización: incertidumbres y potencialidades". X Coloquio de Geografía Rural de España de la Asociación de Geógrafos Españoles. pp. 337-430, 2001.

LARA, J. "Mario Boza, fundador de los parques nacionales: Estamos estafando a los turistas". La Nación, 2017. 
LIPPMANN, J. O. Exacted conservation easements. ProQuest Dissertations and Theses. University of California, 2005.

MELÉNDEZ, C. Costa Rica: Tierra y Poblamiento en la Colonia. San José: Editorial Costa Rica, 1977.

NEL, M. y ANDREU, L. "Organización y características del turismo rural comunitario en Costa Rica”. Anales de Geografía, 28 (2), 167-188, 2008.

NORTHRUP, A. D. The new American conservation movement: New strategies, focus and organizations for the 21 st century. ProQuest Dissertations and Theses. University of Nevada, 2012.

PÉREZ J. D. "Entre La Explotación Y La Conservación De Los Recursos Naturales: El Movimiento Conservacionista Americano En La Segunda Mitad Del Siglo XIX”. Historia Actual Online, n. 1, pp. 57-65, 2003.

PISQUIY, L. Herederas ancestrales y sus demandas actuales Situación de las mujeres indígenas en Centroamérica. San José: UICN, Oficina Regional para Mesoamérica, 2008.

PRATT, L. Logros y retos del turismo costarricense. San José: INCAE, 2002.

QUIRÓS, L. y SÁNCHEZ, A. Volcanes y ecoturismo en México y América Central. Costa Rica: Editorial Universidad Nacional, 2014.

RODRÍGUEZ, G., \& RODRÍGUEZ, A. Problemática agraria de Costa Rica y sus 66 años de historia: 1939-2005. San José, 2007.

ROSENDAL, G. K., y SCHEI, P. J. "How may REDD+ affect the practical, legal and institutional framework for "Payment for ecosystem services" in Costa Rica?". Ecosystem Services, 9,75-82. https://doi.org/10.1016/j.ecoser.2014.04.009, 2014.

SANDNER, G. La colonización agricola de Costa Rica (Instituto). San José, 1964.

SARKAR, S. “Diversity: A philosophical perspective”. Diversity, n. 2, v. 1, pp. 127-41, 2010. https://doi.org/10.3390/d2010127. 
SESNIE, S. E. et al. "A spatio-temporal analysis of forest loss related to cocaine trafficking in Central America”. Environmental Research Letters, n. 12, v. 5, 2017. https://doi. org/10.1088/1748-9326/aa6ff.

SIERRA, R., et al. Patrones y factores de cambio de la cobertura forestal natural de Costa Rica, 1987-2013, (June), 1987-2013. https://doi.org/10.13140/ RG.2.2.33135.74406, 2016.

SINAC. Plan de Acción para la Implementación del Programa de Trabajo sobre Areas Protegidas de la Convención sobre la Diversidad Biológica. San José, 2012.

SINAC. Sistema Nacional de Areas de Conservación. Costa Rica, 2017.

TENORIO, L. A. Las Comunidades indígenas de Costa Rica. San José: Comisión Nacional de Asuntos Indígenas, 1988.

WALLACE, D. The Quetzal and the Macaw: the story of Costa Rica's national parks. San Francisco: Sierra Club Books, 1992. 